\title{
Respuesta histológica completa a la neoadyuvancia en cáncer gástrico avanzado*
}

\author{
Drs. MICHEL PORTANOVA R. ${ }^{1}$, JORGE ORREGO P. ${ }^{1}$, EUGENIO PALOMINO P. ${ }^{2}$ \\ Servicio de Cirugía de Estómago. \\ 2 Departamento de Anatomía Patológica. \\ Hospital Nacional Edgardo Rebagliati. \\ Lima, Perú.
}

\begin{abstract}
\section{Histological complete response after neoadjuvant therapy in advanced gastric cancer}

Introduction: This article describes the first case in Peru where a patient with an advanced gastric cancer showed pathologic complete response to neoadjuvance indicated due an extensive involvement of paraaortic and celiac trunk lymph nodes. Clinical case: 51 year old female patient, after 4 cycles of chemotherapy, the former with epirubicin, cisplatin and capecitabine and the remaining three with epirubicin, cisplatin and 5-fluorouracil, a complete response was obtained from the tomographic point of view. Later patient underwent total gastrectomy with D2 lymphadenectomy. Microscopic examination of the surgical specimen verified only scar tissue without evidence of malignancy in both, the stomach and the lymph nodes removed. Further we present a review of the relevant medical literature to date.

Key words: Gastric cancer, neoadjuvant therapy, complete response.

\section{Resumen}

Introducción: El presente artículo describe el primer caso en el Perú en el que una paciente con cáncer gástrico avanzado ha mostrado respuesta completa histopatológica a neoadyuvancia. Caso clínico: Paciente mujer de 51 años con cáncer gástrico con un extenso compromiso ganglionar para-aórtico y a nivel del tronco celíaco. Luego de 4 ciclos de quimioterapia, el primero con epirrubicina, cisplatino y capecitabina y los 3 restantes con epirrubicina, cisplatino y 5 -fluoruracilo, se obtiene una respuesta completa desde el punto de vista tomográfico, por ello la paciente fue sometida a gastrectomía total con linfadenectomía D2 verificándose en el examen microscópico de la pieza operatoria sólo tejido cicatricial sin evidencias de neoplasia maligna tanto en el estómago como en los ganglios linfáticos resecados. A propósito del caso se hace una revisión de la literatura médica relevante actualizada.

Palabras clave: Cáncer gástrico, terapia neoadyuvante, respuesta completa.

*Recibido el 2 de septiembre de 2012 y aceptado para publicación el 7 de noviembre de 2012.

El presente trabajo no ha tenido apoyo financiero de ningún tipo.

Los autores no declaran conflictos de interés.

Correspondencia: Dr. Michel Portanova R.

Calle Guatemala 165 Dpto. 101 La Molina, Lima 12, Perú.

michelportanova@yahoo.com 


\section{Introducción}

El cáncer gástrico constituye la segunda causa de muerte por cáncer en el mundo ${ }^{1}$, siendo en el Perú la primera $^{2}$. El tratamiento quirúrgico sigue manteniéndose como la piedra angular en el manejo de esta entidad y en tal sentido los resultados mostrados por la escuela japonesa que pone énfasis en la linfadenectomía D2, que entre otros incluye la disección de los ganglios del eje celíaco, han sido claramente superiores. Se reconoce así mismo que el pronóstico de la enfermedad va a estar dado también por el tipo de resección quirúrgica que se realiza, siendo lo más adecuado una resección con intención curativa (R0) en la que no se deja enfermedad residual macro ni microscópica ${ }^{3}$.

Sin embargo, en algunos casos el estudio de extensión de enfermedad previo nos indica la imposibilidad de realizar una cirugía de ese tipo, por ejemplo cuando existe un severo conglomerado ganglionar a nivel del tronco celíaco o a nivel paraaórtico; los pacientes que dentro de ese contexto son sometidos a cirugía muestran una pobre sobrevida en razón que se les termina realizando un procedimiento quirúrgico sub-óptimo ${ }^{4-6}$.

Por otro lado, ya algunos estudios muestran, aunque sin poder establecer conclusiones definitivas, resultados bastante interesantes en relación a neoadyuvancia en el contexto de pacientes con enfermedad altamente avanzada ${ }^{7-9}$.

El presente estudio describe el primer caso reportado en el Perú en el que se verifica una respuesta histopatológica completa luego de neoadyuvancia en una paciente con enfermedad avanzada y compromiso ganglionar extenso realizándose además una revisión de la literatura más relevante acerca de este tópico.

\section{Reporte del caso}

Paciente mujer de 51 años, ingresa al servicio refiriendo una enfermedad de 8 años caracterizada por epigastralgia, 3 meses antes del ingreso se agregan disminución de peso y dispepsia por lo que se realiza una endoscopía digestiva alta.

Endoscopía: Unión esófago-gástrica a los $35 \mathrm{~cm}$, esófago contiene escasos restos hemáticos, pasado el cardias en zona fúndica hacia curvatura mayor lesión tumoral mamelonada e irregular, friable, de $40 \times 40$ $\mathrm{mm}$, resto de fondo normal, cuerpo normal, antro con eritema leve.

Patología: Adenocarcinoma pobremente diferenciado con áreas de células en anillo de sello infiltrante y ulcerado. G3. Viena 5,1 (Figura 1).
Exámenes de laboratorio: Hemoglobina 10,3, grupo sanguíneo A +, CA 19-9: 12,1 U/ml, CEA 0,9 $\mathrm{ng} / \mathrm{ml}$, AFP $27 \mathrm{ng} / \mathrm{ml}$, albúmina 3,53 mg/dl.

Imágenes: Radiografía de pulmones normal. La tomografía (CT) muestra engrosamiento de la pared gástrica en fondo, extenso conglomerado en ligamento gastrohepático e hilio hepático hasta de 6 $\mathrm{cm}$ rodeando el tronco celíaco, y a nivel retroperitoneal en los espacios intercavo-aórtico y para-aórtico izquierdo de $5 \mathrm{~cm}$. No hay líquido libre (Figura 2).

Debido al resultado de la tomografía se transfiere al servicio de Oncología Médica donde se le programa a partir del día 06 de marzo el siguiente esquema terapéutico: Epirrubicina $50 \mathrm{mg} / \mathrm{m}^{2}$ (día 1), Oxaliplatino $130 \mathrm{mg} / \mathrm{m}^{2}$ (día 1), Capecitabina 825 $\mathrm{mg} / \mathrm{m}^{2}$ (día 1 al 14).

Sale de alta el día 08 de marzo pero reingresa por emergencia al día siguiente debido a náuseas, vómitos y epigastralgia que se manejan con tratamiento sintomático e hidratación por 24 h. El día 12 de abril ingresa para su segundo ciclo de quimioterapia en el que se considera pertinente cambiar la capecitabina por 5-Fu debido a la toxicidad mostrada durante el primer curso. La dosis de 5 -Fu fue de $1.000 \mathrm{mg} / \mathrm{m}^{2}$.

El día 15 de mayo ingresa para su tercer ciclo de quimioterapia la que tolera adecuadamente. El día 01 de junio se le realiza un control tomográfico en la que prácticamente no se observan lesiones (Figura 3). El 12 de junio reingresa para su cuarto ciclo de quimioterapia, y debido a la falta de disponibilidad de Epirrubicina recibe en reemplazo Doxorrubicina.

El día 16 de junio se le realiza endoscopía digestiva alta cuyo informe es: unión esófago-gástrica a los $35 \mathrm{~cm}$, en región subcardial hacia curvatura mayor cierta irregularidad de la mucosa de aspecto empedrado, aumentada de consistencia a la toma de biopsia. Resto de estómago con congestión. El resultado de anatomía patológica fue: gastritis crónica superficial y profunda de grado leve con actividad leve, atrofia $+/+++$, sin metaplasia y presencia de dos folículos linfoides reactivos. Helicobacter pylori +/+++, sin células neoplásicas, citokeratina negativa, siendo positiva en células epiteliales (Figura 4). El día 02 de julio se realiza un nuevo control tomográfico verificándose una respuesta completa por lo que se transfiere a cirugía.

Se realiza gastrectomía total radical, linfadenectomía D2 y reconstrucción en Y de Roux con anastomosis esofagoyeyunal manual a puntos separados. Se encuentra ganglio del grupo 8 aumentado de tamaño, consistencia aumentada.

El resultado de anatomía patológica fue el siguiente: macroscopía muestra serosa lisa, congestiva, abierto el espécimen por curvatura mayor se observa en cuerpo alto cara anterior a $2 \mathrm{~cm}$ del borde 


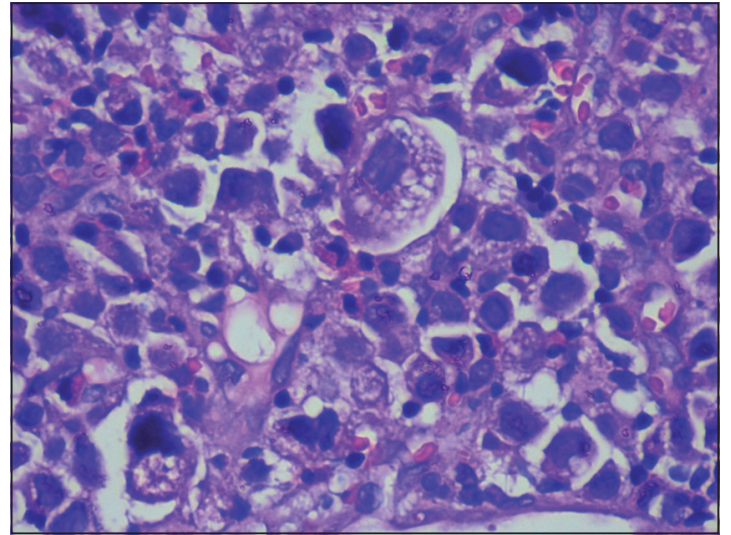

Figura 1. Microfotografía a gran aumento mostrando la biopsia inicial, correspondiente a Carcinoma indiferenciado, con aisladas células en anillo de sello, infiltrante.

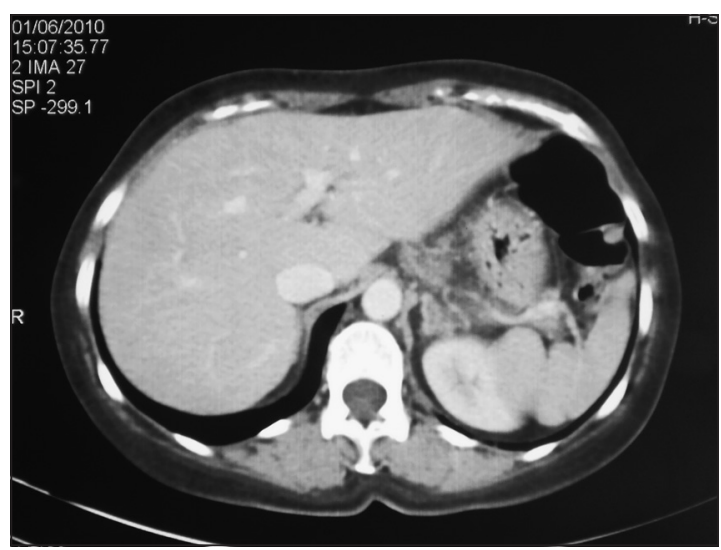

Figura 3. Tomografía luego de 3 ciclos de terapia neo adyuvante que muestra estómago pequeño con respuesta casi completa del compromiso ganglionar previo

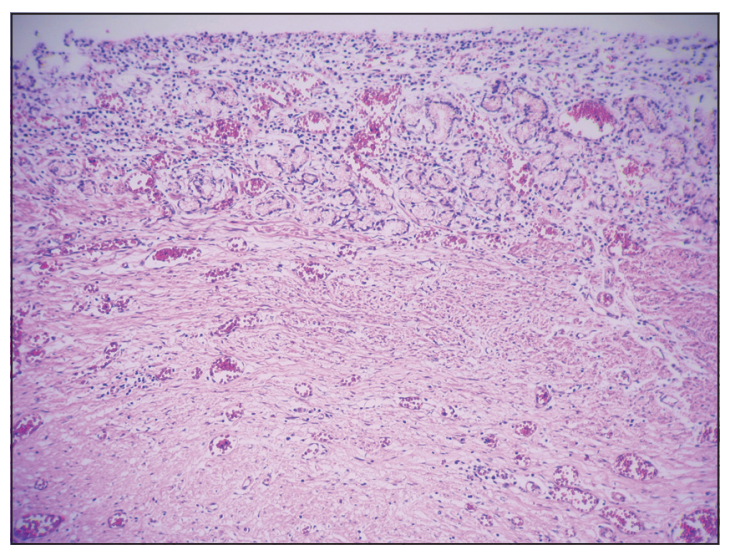

Figura 5. Microfotografía a mediano aumento de la lesión cicatricial residual en espécimen de gastrectomía. Además de lo anteriormente descrito, se observa vasos sanguíneos de aspecto reparativo.

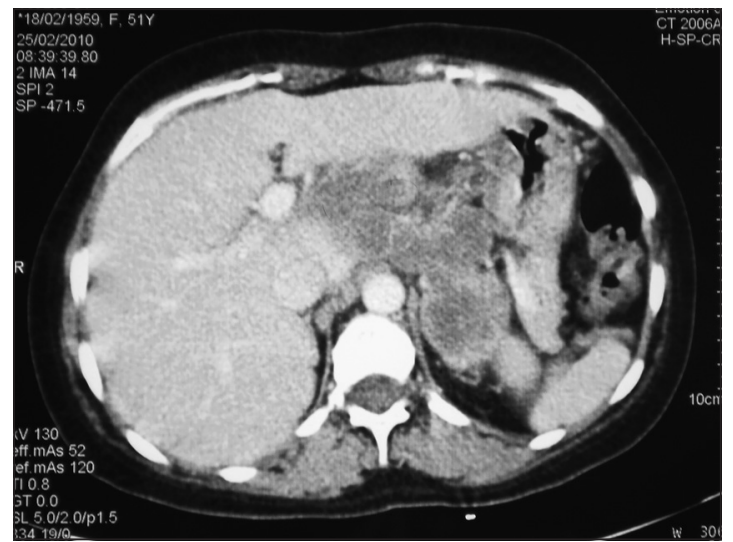

Figura 2. Tomografía de estadiaje que muestra compromiso ganglionar grosero en territorio intercavo-aórtico, para-aórtico izquierdo y del tronco celíaco.

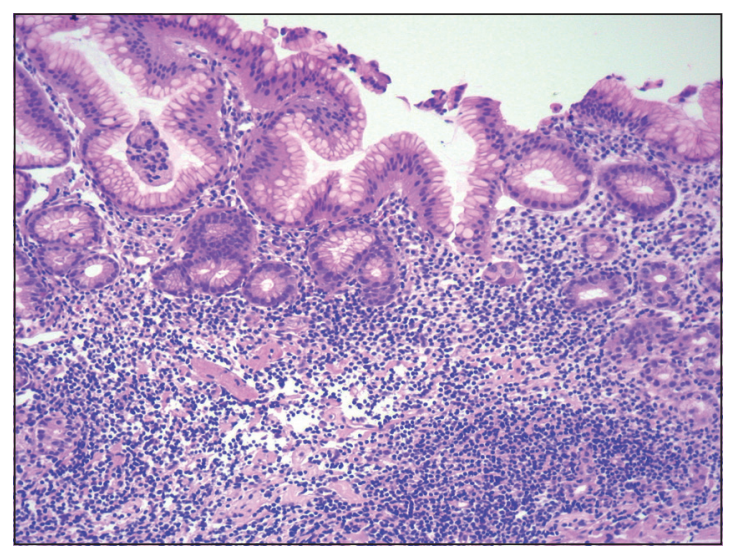

Figura 4. Microfotografía a mediano aumento de la biopsia de control post tratamiento. Se observa gastritis crónica moderada inespecífica y discreta hiperplasia foveolar. No se observa neoplasia maligna.

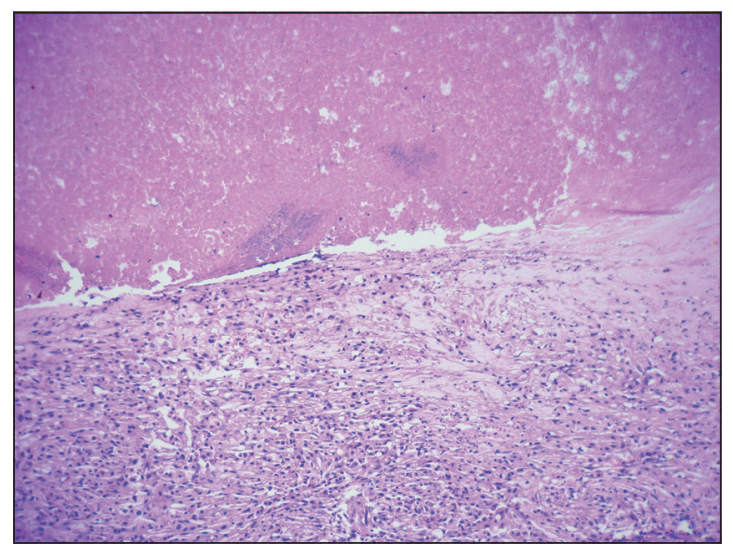

Figura 6. Microfotografía a mediano aumento de ganglio linfático del grupo 8-9. Se observa necrosis en la mitad superior del campo y fibrosis e inflamación crónica inespecífica en la mitad inferior. No se identifica neoplasia. 
esofágico una lesión cicatricial lineal de $3 \mathrm{~cm}$ de eje mayor longitudinal con centro deprimido, de bordes regulares y consistencia elástica. Microscopía: lesión cicatricial lineal de $3 \mathrm{~cm}$ en cuerpo alto sobre cara anterior, sin neoplasia maligna residual, con cambios epiteliales y vasculares relacionables a terapia oncológica. Gastritis crónica moderada con grupos de histiocitos murales. Ganglios linfáticos negativos para neoplasia maligna (Figuras 5 y 6 ).

\section{Discusión}

El cáncer gástrico es la cuarta neoplasia más frecuente en el mundo y la segunda causa de muerte por cáncer ${ }^{1}$. En el Perú es la primera causa de muerte por cáncer ${ }^{2}$. La posibilidad de sobrevivir a la enfermedad está directamente relacionada al estadío, así en los casos de cáncer temprano las sobrevida luego de tratamiento quirúrgico o inclusive endoscópico está por encima del $90 \%{ }^{10,11}$, sin embargo, sólo en países orientales como Korea y Japón las tasas de detección de cáncer temprano se mueven por encima del 35 y $50 \%$ respectivamente ${ }^{12,13}$, entre otros, debido a políticas gubernamentales agresivas. Sin embargo, en occidente ocurre todo lo contrario porque la norma sigue siendo detectar la enfermedad en estadíos avanzados ${ }^{14}$, lo que disminuye la efectividad del tratamiento.

En el contexto del cáncer avanzado de estómago la técnica quirúrgica japonesa (linfadenectomía D2) es la que ha mostrado los mejores resultados, siempre y cuando se logre el concepto de cirugía con intención curativa a través de una resección tipo $\mathrm{R} 0$, es decir, donde no se deje enfermedad residual macro ni microscópica. Sin embargo, aún con una buena cirugía el pronóstico de muchos pacientes con estadío clínico III y IV no es de los mejores.

Por otro lado, el desarrollo incesante de las técnicas de imágenes para el estudio de extensión de la enfermedad, nos permite cada vez en una mayor medida, tener un vislumbre de aquellos pacientes que tienen factores que van a imposibilitar una cirugía tipo R0 ya sea por enfermedad secundaria hepática, signos en relación a carcinomatosis o por un compromiso ganglionar regional grosero. En este escenario la cirugía resectiva podría tener algún rol, pero básicamente mejorando la calidad de vida y prolongando algo la sobrevida, sin embargo, el desarrollo de nuevos agentes quimioterápicos y el énfasis en el manejo multimodal del cáncer gástrico, sobretodo en occidente, abre la posibilidad de intentar estrategias distintas de manejo dentro del contexto antes anotado, y sólo dentro de ese contexto, ya que la neoadyuvancia en el escenario de la enfermedad que se presume primariamente resecable con intención curativa, aún se encuentra caminando en el terreno de lo experimental.

Así tenemos que existen reportes desde fines de los ochenta donde se describen un $45 \%$ de resección luego de quimioterapia (etopósido, doxorrubicina y cisplatino) en pacientes primariamente considerados irresecables encontrándose sólo en un tercio de ellos una respuesta patológica completa ${ }^{15}$.

Con ECF (epirrubicina, cisplatino, 5-fluoruracilo), Melcher ${ }^{16}$, a mediados de los noventa reporta en los pacientes primariamente irresecables sólo $10 \%$ de resecabilidad. Ningún caso con respuesta patológica completa. Incluye un total de 27 pacientes con cáncer de estómago y de esófago inferior. $37 \%$ de pacientes fueron considerados primariamente irresecables por CT y por laparotomía. No en todos se documentó CT pero en los 20 que se documentó $60 \%$ tuvieron respuesta.

También con ECF, un estudio de $\mathrm{Geh}^{17}$ del año 2000 sobre 23 pacientes reporta $4,3 \%$ de respuesta patológica completa, sin embargo, el estudio incluye pacientes tanto potencialmente resecables como irresecables y de la unión esófago gástrica como de estómago. En ninguno de los 4 pacientes considerados a priori irresecables se pudo conseguir una cirugía resectiva.

Los últimos 2 estudios antes señalados revelan falta de estandarización en el proceso de estadiaje preoperatorio el que además no incluye laparoscopía y no hay un análisis detallado de subgrupos entre los tumores primariamente resecables e irresecables.

Interesantemente en el Japón a finales de los noventa Nashimoto $^{18}$, presenta los resultados de su estudio de fase II sobre 21 pacientes en el cual se estandariza la población incluyendo sólo a aquellos con tumores irresecables y a aquellos que siendo potencialmente resecables tenían factores de incurabilidad, usa FLP (5-Fluoruracilo, leucovorina y cisplatino) No se reportó respuesta completa pero sí parcial en el $57 \%$ y para los ganglios para aórticos fue de $64 \%$, mejorándose la resecabilidad en el grupo que respondieron.

En el 2000 Gallardo-Rincón ${ }^{19}$ en México, reporta un estudio similar de fase II con P-ELF (cisplatino, etopósido, leucovorina, 5-fluoruracilo) en una de las series más amplias sobre 60 pacientes y estandarizando también el grupo de pacientes sólo a aquellos con cáncer gástrico irresecable, sin embargo, no se establece cuales eran los criterios para declararlos irresecables, sólo incluye estadios III y IV desde el punto de vista clínico. Desde el punto de vista tomográfico en 33\% hubo respuesta parcial, siendo ésta más evidente en el grupo de pacientes de estadío III y en sólo un paciente hubo respuesta completa. Sólo 10 pacientes $(16,5 \%)$ pudieron ser resecados, y de 
ellos sólo 5 con intención curativa. Sugiere como conclusión un estudio fase III.

En el año 2004 GISCAD, que es el grupo italiano para el estudio del cáncer de tracto digestivo, utilizando el esquema PELF (cisplatino, epidoxorrubicina, leucovorina y 5 -fluoruracilo) reporta $49 \%$ de respuesta entre parcial y completa desde el punto de vista tomográfico, con $5 \%$ de respuesta completa histopatológica ${ }^{20}$. El estudio incluye sólo pacientes irresecables pero en el $63 \%$ de pacientes se estableció esta condición luego de laparotomía.

Hacia el año 2006 Mori $^{2}$ en el Japón usando S-1, droga que hoy es considerada de uso estándar en adyuvancia postoperatoria para los estadíos II y III, reporta un interesantísimo caso en el que se consigue respuesta completa patológica en un paciente con estadío clínico T2, N0, M0, perfectamente resecable pero que decidió diferir su cirugía por un mes ofreciéndosele neoadyuvancia durante ese período, la cual aceptó.

En el mismo año $\mathrm{D}^{\prime} \mathrm{Ugo}^{22}$ en Italia, utiliza EEP (etopósido, epirrubicina y cisplatino) como neoadyuvancia antes de cirugía D2. Reporta respuesta patológica completa en 1 caso $(3,3 \%)$. Este estudio sobre 30 pacientes resulta muy interesante porque se incluye laparoscopía dentro del proceso metodológico y además citología, lo cual ya constituye un detalle valioso si partimos del hecho que la siembra peritoneal es mucho más resistente al tratamiento, asímismo se incluyen básicamente pacientes T3 y T4 y sólo 4 pacientes T2 pero con evidente compromiso ganglionar verificado en la laparoscopía. Llama la atención que el T se estableció sólo visualmente a la laparoscopía y no se detalla cuantos correspondían a T3 y T4, esto es importante porque aunque se reporta $43 \%$ de regresión del $\mathrm{T}$ basado en criterios anatomopatológicos, en realidad no sabemos cuántos de los que visualmente impresionaron como T3 en realidad no lo eran, así mismo no se define cuantos redujeron por ejemplo de T4 a T3 evitándose una resección ampliada a órganos vecinos comprometidos. Se logró resección R0 en el $80 \%$ de los casos, pero no se establece si los casos eran primariamente resecables o no.

En el 2007 Bueno Muiño ${ }^{23}$ en España, usando el esquema TCF (docetaxel, cisplatino, 5-fluoruracilo) reporta un caso de respuesta patológica completa luego de 4 ciclos, llama la atención que la irresecabilidad del tumor se verificó en la laparotomía exploratoria aún cuando la masa tumoral y el compromiso ganglionar eran groseros en la tomografía.

En el 2010 Biffi $^{24}$ en Italia, reporta su estudio en el que utiliza también el esquema TCF (docetaxel, cisplatino, 5-fluoruracilo) en 35 pacientes e incluye un brazo de otros 35 pacientes que van a cirugía directamente. Reporta $11,7 \%$ de respuesta patológica completa y $55 \%$ de respuesta patológica parcial, incluye pacientes T3-4 independientemente del N y cualquier $\mathrm{T}$ con $\mathrm{N}$ positivo, incluye laparoscopía previa pero no citología. Al igual que lo ocurrido en el estudio de D'Ugo, lo interesante sería saber cuántos pacientes reducen su $\mathrm{T}$ y no sólo que tipo de respuesta tiene el tumor desde el punto de vista patológico. Adicionalmente, aunque no se especifica el porcentaje de pacientes que hicieron progresión en el grupo de neoadyuvancia, se verifica $85 \%$ de resección R0 para el grupo de neoadyuvancia y $91 \%$ para el otro grupo.

Resultados como los mostrados en estos reporte son estimulantes y abren nuevos senderos dentro del desarrollo de estrategias multimodales de manejo del cáncer gástrico avanzado, sin embargo, deben ser tomados con cautela y rigor científico, estimulando el desarrollo de protocolos de investigación que nos permitan definir tanto los grupos de pacientes a ser beneficiados y el esquema terapéutico más adecuado.

En cuanto al esquema terapéutico a usar, dada la experiencia en el Japón con el uso de S1, que dicho sea de paso es la droga estándar en adyuvancia postoperatoria, probablemente sea una buena opción a manejar a futuro, sin embargo, en occidente no sólo es poco usada sino que el principal problema es la gran cantidad de esquemas terapéuticos que se disponen. A la luz de los estudios antes mencionados tal vez las mejores opciones sean la combinación de 5-fluoruracilo, cisplatino y epirrubicina o docetaxel.

Los aspectos a tener cuidado cuando se intente neoadyuvancia incluyen una adecuada homogenización de pacientes, en ese sentido aquellos pacientes potencialmente resecables de primera intención y en donde se prevé que una cirugía con intención curativa puede ser perfectamente realizada probablemente no sean nuestra población objetivo. Creemos que un buen punto de partida para esta alternativa de manejo multimodal podrían ser pacientes irresecables debido a compromiso local extenso y pacientes potencialmente resecables pero con conglomerados para-aórticos o del eje celíaco que imposibiliten una resección R0, teniendo especial cuidado en la inclusión de laparoscopía y citología de rutina que son fundamentales para evaluar adecuadamente la respuesta en este tipo de pacientes.

\section{Referencias}

1. Parkin DM, Bray F, Ferlay J, Pisani P. Global cancer statistics. 2002 Cancer J Clin. 2005;55:74-108.

2. Mendoza D, Herrera P, Gilman R, Lanfranco J, Tapia M, Busalleu A, et al. Variation in the prevalence of gastric cancer in Perú. Int J Cancer. 2008;123:414-20. 
3. Roukos DH, Lorenz M, Karakostas K, Paraschou P, Batsis C, Kappas AM. Pathological serosa and nodebased classification accurately predicts gastric cancer recurrence risk and outcome, and determines potential and limitation of a japanese-style extensive surgery for western patients: A prospective with quality control 10year follow-up study. BJC 2001;84:1602-9.

4. Saidi R, Remine S, Dudrick P, Hanna N. Is there a role for palliative gastrectomy in patients with stage IV gastric cancer? World J Surg. 2006;30:21-7.

5. Kunisaki CH, Makino H, Takagawa R, Oshima T, Nagano Y, Fujii S, et al. Impact of palliative gastrectomy in patients with incurable advanced gastric cancer. Anticancer Res. 2008;28:1309-16.

6. Miyagaki H, Fujitani K, Tsujinaka T, Hirao M, Yasui M, Kashiwazaki M, et al. The significance of gastrectomy in advanced gastric cancer patients with non-curative factors. Anticancer Res. 2008;28:2379-84.

7. Nashimoto A, Yabusaki H, Nakagawa S, Takii Y, Tsuchiya Y, Otsuo T. Preoperative chemotherapy with s-1 and cisplatin for highly advanced gastric cancer. Anticancer Res. 2009;29:4689-96.

8. Kochi M, Fujii M, Kanamori N, Kaiga T, TakahashI T, Kobayashi M, et al. Neoadjuvant chemotherapy with S-1 and CDDP in advanced gastric cancer. J Cancer Res Clin Oncol. 2006;132:781-5.

9. Yoshikawa T, Sasako M, Yamamoto S, Sano T, Imamura H, Fujitani K, et al. Phase II study of neoadjuvant chemotherapy and extended surgery for locally advanced gastric cancer. Br J Surg. 2009;96:1015-22.

10. Fuji M, Sasaki J, Nakajima T. State of the art in the treatment of gastric cancer: from the $71^{\text {st }}$ Japanese gastric cancer congress. Gastric Cancer1999;2:151-7.

11. Gotoda T. Endoscopic resection of early gastric cancer: the Japanese perspective. Curr Opin Gastroenterol. 2006;22:561-9.

12. Lee JH, Han SH, Lee JH. A prospective randomized study comparing open vs. laparoscopy-assisted distal gastrectomy in early gastric cancer. Early results. Surg Endosc. 2005; 19:168-73.

13. Ono H. Early gastric cancer: diagnosis, pathology, treatment techniques and treatment outcomes. Eur J Gastroenterol Hepatol. 2006;18:863-6.

14. Portanova M, Vargas F, Lombardi E, Mena V, Carbajal $\mathrm{R}$, Palacios N, et al. Results of specialization in the surgical treatment of gastric cancer in Peru. Gastric Cancer 2007;10:92-7.

15. Wilke H, Preusser P, Fink U, Gumzer U, Meyer HJ,
Siewert JR, et al. Preoperative chemotherapy in locally advanced and non resectable gastric cancer: a phase II study with etoposide, doxorubicin and cisplatin. J Clin Oncol. 1989;7:1318-26.

16. Melcher AA, Mort D, Maughan DS. Epirubicin, cisplatin and continuous infusion 5-fluorouracil (ECF) as neoadjuvant chemotherapy in gastro-oesophageal cancer. BJC 1996;74:1651-4.

17. Geh J, Glynne-Jones R, Kwok Q, Banerji U, Livingstone J, Townsend E, et al. Preoperative ECF Chemotherapy in Gastro-Oesophageal Adenocarcinoma. Clin Oncol. 2000;12:182-7.

18. Nashimoto A, Yabusaki H, Tanaka O, Sasaki J, Akiyama N. Neoadjuvant chemotherapy in advanced gastric cancer with non curative factors: a phase II study with 5-fluoruracil, leucovorin and cisplatin. Gastric Cancer 1999;2:57-63.

19. Gallardo-Rincon D, Oñate-Ocaña L, Calderillo-Ruiz G. Neoadjuvant chemotherapy with P-ELF (cisplatin, etoposide, leucovorin, 5-fluorouracil) followed by radical resection in patients with initially unresectable gastric adenocarcinoma: a phase II study. Ann Surg Oncol. 2000; 7:45-50.

20. Cascinu S, Scartozzi M, Labianca R, Catalano V, Silva RR, Barni S, et al. High curative resection rate with weekly cisplatin, 5-fluorouracil, epidoxorubicin, 6Sleucovorin, glutathione, and filgastrim in patients with locally advanced, unresectable gastric cancer: a report from the italian group for the study of digestive tract cancer (GISCAD). BJC 2004;90:1521-5.

21. Mori S, Kishimoto H, Tauchi K, Higuchi K. Histological complete response in advanced gastric cancer after 2 weeks of S-1 administration as neoadjuvant chemotherapy. Gastric Cancer 2006;9:136-9.

22. D’Ugo, Persiani R, Rausei S, Biondi A, Vigorita V, Boccia S, et al. Response to neoadjuvant chemotherapy and effects of tumor regression in gastric cancer. EJSO 2006;32:1105-9.

23. Bueno Muiño C, Puente Vázquez J, Sastre Valera J, García-Sáenz JA, Martin M, García Miralles N, et al. Pathological complete response following docetaxelbased neoadjuvant chemotherapy for locally advanced gastric adenocarcinoma. Clin Transl Oncol. 2007;9:3358 .

24. Biffi R, Fazio N, Luca F, Chiappa A, Andreoni B, Zampino MG, et al. Surgical outcome after docetaxel-based neoadjuvant chemotherapy in locally-advanced gastric cancer. World J Gastroenterol. 2010;21:868-74. 